\title{
BRDF Slices: Accurate Adaptive Anisotropic Appearance Acquisition
}

\author{
Jiří Filip ${ }^{1}$ Radomír Vávra ${ }^{1}$ Michal Haindl $^{1} \quad$ Pavel Žid $^{1} \quad$ Mikuláš Krupička $^{1}$ Vlastimil Havran ${ }^{2}$ \\ ${ }^{1}$ Institute of Information Theory and Automation of the AS CR, Prague \\ ${ }^{2}$ Faculty of Electrical Engineering, Czech Technical University in Prague \\ \{filipj, vavra, haindl, zid,krupimik\}@utia.cas.cz, havran@fel.cvut.cz
}

\begin{abstract}
In this paper we introduce unique publicly available dense anisotropic BRDF data measurements. We use this dense data as a reference for performance evaluation of the proposed BRDF sparse angular sampling and interpolation approach. The method is based on sampling of BRDF subspaces at fixed elevations by means of several adaptively-represented, uniformly distributed, perpendicular slices. Although this proposed method requires only a sparse sampling of material, the interpolation provides a very accurate reconstruction, visually and computationally comparable to densely measured reference. Due to the simple slices measurement and method's robustness it allows for a highly accurate acquisition of BRDFs. This in comparison with standard uniform angular sampling, is considerably faster yet uses far less samples.
\end{abstract}

\section{Introduction}

Accurate acquisition and representation of real-world materials' appearance is of an ultimate challenge in computer vision and graphics. Although many factors influence the appearance of materials, we investigate in this paper those which are most apparent - illumination and view directions. A directional properties of material reflectance was formalized by Nicodemus et al. [15] in a form of a bidirectional reflectance distribution function $\operatorname{BRDF}\left(\lambda, \theta_{i}, \varphi_{i}, \theta_{v}, \varphi_{v}\right)$, where $\lambda$ is light wavelength or color spectrum, and the remaining parameters represent illumination $\omega_{i}=\left[\theta_{i}, \varphi_{i}\right]$ and view $\omega_{v}=\left[\theta_{v}, \varphi_{v}\right]$ directions, each parameterized by elevation $\theta$ and azimuth $\varphi$ angles as shown in Fig. 1. In this paper a novel BRDF goniometric

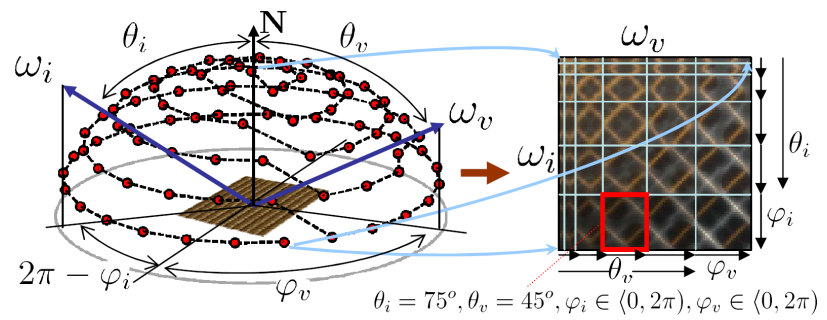

Figure 1. A parameterization of illumination and view directions. measurement setup is introduced and its very dense BRDF measurements are analyzed. Additionally, we introduce a novel adaptive method of highly accurate interpolation of sparsely measured BRDF and have made measured data publicly available for research purposes.

\section{Related Work}

Based on the way the four degrees of mechanical freedom are realized, BRDF acquisition setups can be divided into three categories. The first is based on gonioreflectometers where all combinations of illumination and viewing directions are achieved by the sequential mutual positioning of light, sensor, and sample [7, 8]. The second category leverages mechanical complexity using parabolic mirrors to capture multiple views in a single image [1, 4]. The third category utilizes a prior knowledge of non-planar sample geometry to capture in a single image many different illumination and viewing directions. These setups capture either isotropic [12] or anisotropic [10, 11, 14] BRDF data. The last two categories have either compromised accuracy, as well as limitations in the effective range of elevation angles, or require certain geometry of the sample making the measurement procedure more efficient.

Although four anisotropic BRDFs have been made available for research purposes [14], their minimal azimuthal sampling step is $2^{\circ}$ only and the resulting data are extremely noisy. Therefore, we introduce a novel gonioreflectometerbased acquisition setup for measurement of anisotropic BRDF with unique angular density and accuracy. Such precise measurement data are then used as reference data for the proposed interpolation algorithm evaluation.

General methods of adaptive sampling [18] have been extensively studied. Their application to adaptive illumination sampling was investigated in [3]. Robinson and Ren [16] applied sparse one-dimensional yard-stick coding for image data compression along ridges and valleys obtained using a Laplacian-of-Gaussian image filter. They proposed a suboptimal relaxation algorithm for refinement of sparse samples positions. Sampling of known 1D functions based on Douglas-Peucker algorithm was introduced in [9]. How- 
ever, these approaches assume prior knowledge of the entire data, while we use only a sparse adaptive sampling of unknown reflectance data constrained by a typical BRDF behavior, i.e., we sequentially measure new samples to continually improve overall reconstruction. To date, we are not aware of any literature on a sparse adaptive acquisition of unknown 4D BRDFs.

Our work is motivated by the method of Filip and Vávra [2], using a sparse set of azimuthal slices placed orthogonally to main reflectance features in BRDF space. These slices allow for an efficient and robust reconstruction.

\section{Measurement Setup}

All the measurements were done using the gonioreflectometer shown in Fig. 2 .

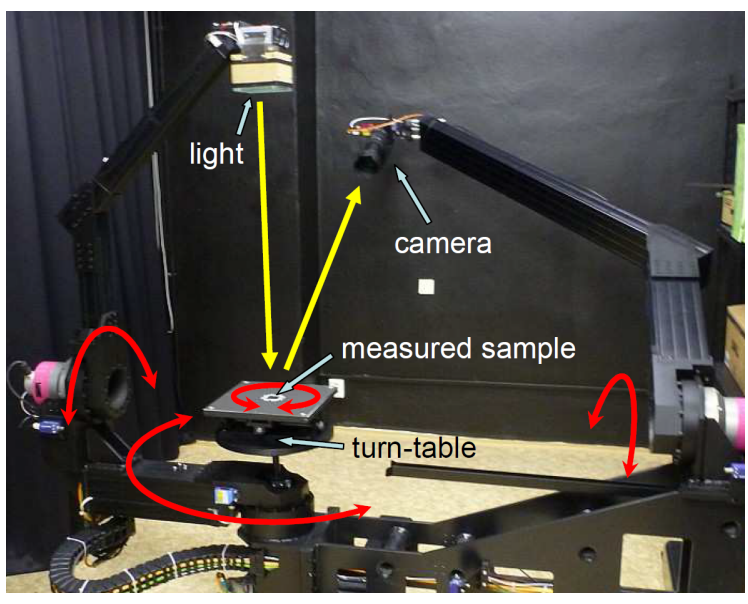

Figure 2. The UTIA gonioreflectometer.

Mechanical Construction - The setup consists of the measured sample held by a rotating stage and two independently controlled arms with camera (one axis) and light (two axes) as shown in Fig. 2. It allows for flexible and adaptive measurements of nearly arbitrary combinations of illumination and viewing directions. Although camera view occlusion by arm with light may occur, it can be analytically detected, and in most cases alternative positioning is possible. Verified illumination and camera arms positioning angular accuracy across all axes is $\pm 0.03^{\circ}$.

Illumination Source - Due to advantages of color stability, long-lifetime and reasonable heat dissipation, we used a custom build array of 11 LED Cree XML, each having flux $280 \mathrm{~lm}$ at $0.7 \mathrm{~A}$ (max. current $3 \mathrm{~A}$ ). Each LED is equipped with its own optics producing a narrow and uniform beam of light. HDR acquisition is achieved by adaptive exposure times and variable lighting intensity (through current being fed into LEDs); both of this is controlled remotely depending on the dynamic range of the measured sample.

Imaging Device - An industrial full-frame 16Mpix RGB camera AVT Pike 1600C was used in the setup containing a Kodak KAI-16000 CCD sensor (14 bits/channel), resolution $4872 \times 3248$ pixels and the shortest integration time of $0.6 \mathrm{~ms}$. The sensor's distance from the sample was $\approx 2 \mathrm{~m}$. Using two different optics we achieved two spatial resolutions: 350 DPI (i.e., $73 \mu \mathrm{m} /$ pixel) and 1071 DPI (i.e., $24 \mu \mathrm{m} / \mathrm{pixel})$, which constrained maximal sample's size to $140 \times 140 \mathrm{~mm}$ and $44 \times 44 \mathrm{~mm}$, respectively.

System Calibration and Data Processing - Zero initial positions of all axes were found using a spirit level, plummet, and device's axes intersection, etc. Lighting nonuniformity over the target area was measured using a luxmeter, fitted by a 2D polynomial, and compensated from the photos. The camera's defective pixels were detected and interpolated in the photos. Internal Bayer pattern in the raw float data is interpolated into RGB using a local linear interpolation. Optics vignetting is estimated from photo of a white sheet and its non-uniformity is compensated. A colorimetric calibration $3 \times 3$ matrix is obtained by solving a set of linear equations relating known and measured color patches on Xrite target in CIE XYZ colorspace. Images taken in the OpenEXR floating point HDR format from different views are mutually pixel-wisely registered by means of lines detection on registration target surrounding the measured object.

System Control - The complete measurement procedure is automatically controlled by a single server. The control application stores the list of the required measured positions, which can be adaptively modified during measurement. Measured data are stored on a 20 terabytes disk-array and accessed via speedy 10 Gbit optical LAN. Mechanical positioning, exposure, and data transfer of 6561 measurements typically took around 18 hours.

\section{Uniform Sampling Evaluation}

View- and illumination-dependent reflectance properties of materials are often measured uniformly over a hemisphere. As examples can serve $81 \times 81$ directions sampling (6561 samples) [17] or $151 \times 151$ directions sampling (22801 samples) [13]. Unfortunately, even such relatively dense sampling is insufficient to accurately capture important reflectance behavior. Fig. 3 compares visualization quality of five materials when the ground truth data and uniform sampling $151 \times 151$ directions are used. To obtain ground truth data we rendered a sphere in resolution $128 \times 128$ pixels using a simple ray tracer implemented in Matlab and generated a list of 10975 view and illumination directions, one for each sphere pixel. Then all these directions were measured using the gonioreflectometer and visualized.

As expected, even with a high number of uniform samples the differences are significant, especially for specular materials. Therefore, we investigate alternative means of 


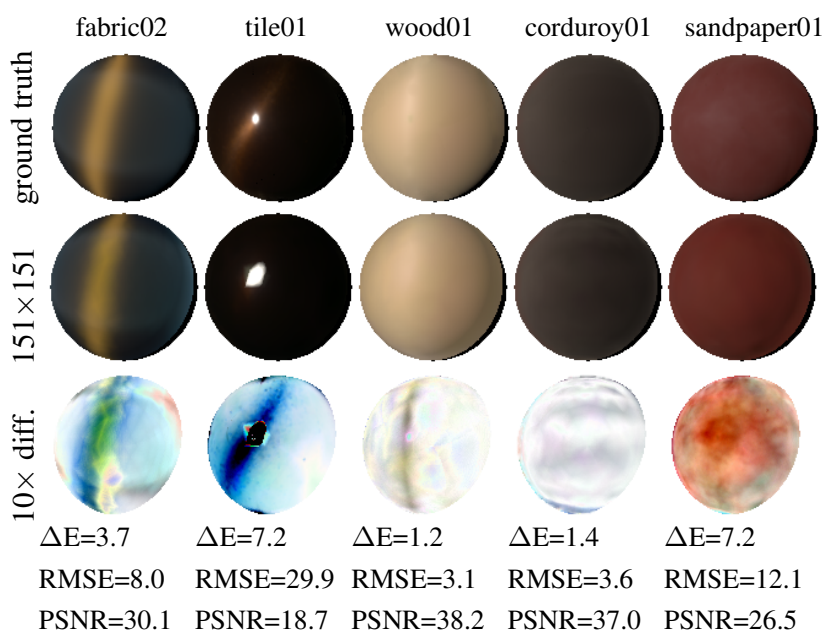

Figure 3. A comparison of BRDF visualization on a sphere: measured 10975 exact directions in each pixel (the first row) vs. uniformly measured and interpolated $151 \times 151$ directions (the second row). Below are the $10 \times$ difference images (the third row) and CIE Lab $\triangle \mathrm{E} / \mathrm{RMSE} / \mathrm{PSNR}[\mathrm{dB}]$ values.

view- and illumination-dependent material appearance sampling to achieve better reconstruction precision using less samples.

\section{A Test Sample Measurement and Analysis}

Even an exhaustive measurement of all combinations of view and illumination directions in $1^{\circ}$ step would require $(360 \times 90)^{2} \approx 10^{9}$ measurements, the acquisition time using our setup would exceed reasonable limits $(\approx 329$ years $)$ and still would not guarantee ideal measurements. Therefore, we focused first on a dense measurement of BRDF subspace for fixed elevations of a single but challenging material. Our goal is to investigate the adaptive sparse sampling algorithm which can represent appearance of this subspace very precisely using a reasonable number of samples.

We selected fabric material consisting of two perpendicular finely interwoven yellow and gray fibers (see Fig. 4 topleft). This material provides an intricate golden appearance with a strong anisotropic behavior as shown in example images of various illumination and viewing conditions in the second row of Fig. 4

When the material's BRDF was measured uniformly in $81 \times 81$ and $151 \times 151$ sampling, we obtained the result illustrated in the first row of Fig. 4 (see Fig. 1 for values ordering explanation). Visualization of this BRDF on a sphere for $151 \times 151$ sampling is shown in the second row of Fig. 3-left. Note that the minimal azimuthal distance of individual samples in this data is $15^{\circ}$. In the further experiment we selected BRDF subspace at the highest elevation angles exhibiting the strongest anisotropic behavior, i.e., $\theta_{i}=\theta_{v}=75^{\circ}$ (see Fig. 4 top-right). Figure 5 compares the reference subspace measurement (left) with two

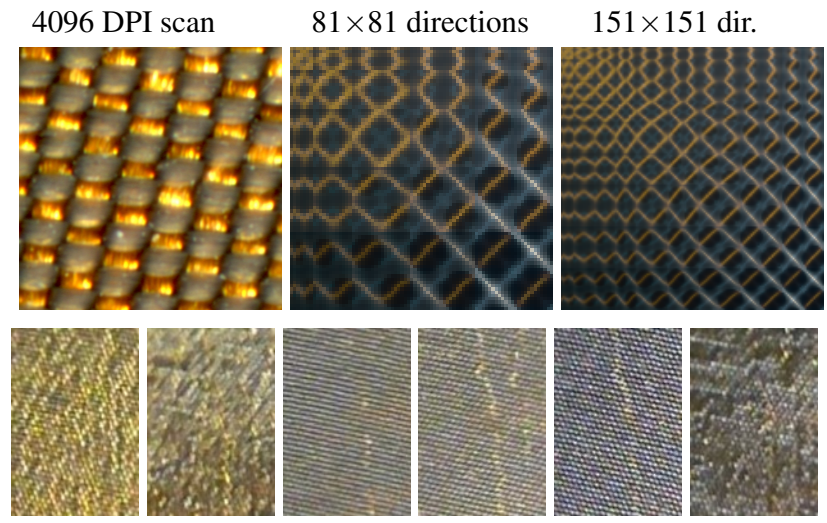

Figure 4. The first row: High resolution scan of the fabric material (left), its uniformly measured BRDF: in $81 \times 81$ (middle) and $151 \times 151$ (right) directions. The second row: Examples of material's appearance for different illumination and view conditions.
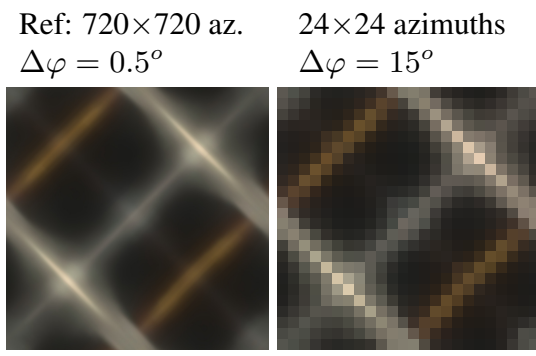

$48 \times 48$ azimuths $\Delta \varphi=7.5^{\circ}$

Figure 5. A BRDF subspace for view and illumination elevation $75^{\circ}$. Densely measured reference data with samples' distance $\Delta \varphi=0.5^{\circ}$ (left); sparsely measured - samples' distance $\Delta \varphi=15^{\circ}$ (middle) and $\Delta \varphi=7.5^{\circ}$ (right). The middle image corresponds to sampling $81 \times 81$ and $151 \times 151$.

uniform azimuthal sampling densities (middle, right). The reference data are densely measured using azimuthal sampling step $\approx \Delta \varphi=2^{\circ}$ using 11856 samples and interpolated into a resolution $\Delta \varphi=0.5^{\circ}$ (left). The subspace measured using 24 azimuthal samples ( $\operatorname{step} \Delta \varphi=15^{\circ}$ ) contains $n=\left(360^{\circ} / 15^{\circ}\right)^{2}=576$ samples (middle), and the subspace measured using 48 azimuthal samples $\left(\Delta \varphi=7.5^{\circ}\right)$ contains $n=\left(360^{\circ} / 7.5^{\circ}\right)^{2}=2304$ samples (right). Performance of a barycentric interpolation of these uniform samples into reference data resolution $\left(\Delta \varphi=0.5^{\circ}\right)$ is shown in the second and fourth column of Fig. 10 These results prove unsatisfactory performance of the uniform acquisition approaches.

\section{Sparse Data Acquisition and Interpolation}

Proposed sparse data acquisition and interpolation is similar to [2], where based on two perpendicular slices measured across azimuthal angles and fixed light and camera elevations, a BRDF subspace parametrization and interpolation was proposed, as shown in Fig. 6 . The slice aligned with the direction of specular highlights is called axial slice 


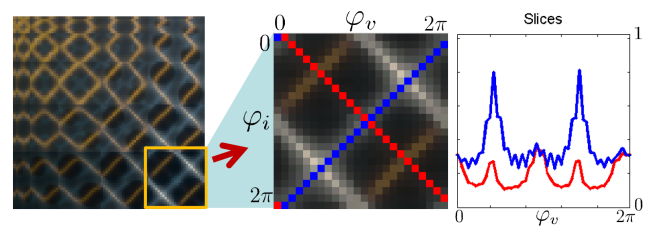

Figure 6. A schema of a subspace interpolation using two slices.

$s_{A}$ (red), i.e., $\varphi_{v}-\varphi_{i}=\alpha$ holds for azimuthal angles. The axial slice records the material's anisotropic properties (mutual azimuthal position of the light and camera is fixed while the sample rotates), i.e., for near-isotropic samples it is almost a constant value. The slice perpendicular to the highlights is called diagonal slice $s_{D}$ (blue), i.e., $\varphi_{i}+\varphi_{v}=2 \pi+\beta$ holds for azimuthal angles. The diagonal slice captures the shape of the specular peaks (light and camera travel in mutually opposite azimuthal directions over the sample). Both slices can be expressed as

$s_{A, \theta_{i}, \theta_{v}, \alpha}\left(\varphi_{v}\right)=\operatorname{BRDF}\left(\theta_{i}, \varphi_{i}=\varphi_{v}-\alpha, \theta_{v}, \varphi_{v}\right)$,

$s_{D, \theta_{i}, \theta_{v}, \beta}\left(\varphi_{v}\right)=\operatorname{BRDF}\left(\theta_{i}, \varphi_{i}=2 \pi-\varphi_{v}+\beta, \theta_{v}, \varphi_{v}\right)$.

While [2] focuses on approximative subspace reconstruction using two slices only, we attempt for very accurate reconstruction of the subspace using the set of 12 axial and 12 diagonal slices. The slices' placement is realized uniformly across the subspace in azimuthal step $30^{\circ}$. Such a placement divides the subspace image into a grid of rectangles (see Fig. 10-f). The slices values are adaptively measured; however, the remaining data has to be interpolated. Section 6.1 explains method of adaptive sampling along slices and Section 6.2 describes method of slices values propagation to missing parts of the BRDF subspace.

\subsection{Adaptive Slice Sampling}

Each slice can be interpreted as a one dimensional periodic signal with period $360^{\circ}$. As such, it can be sampled uniformly with a defined step (e.g., $1^{\circ}$ ) or adaptively decreasing a number of samples on the one hand and increasing reconstruction accuracy in areas with high variance on the other. As the behavior of the signal is unknown, the adaptive algorithm can work with already measured samples only; adding new samples in areas where it can improve reconstructed signal accuracy.

We propose the following algorithm outlined in Fig. 7 . left, which can be viewed as a form of cross-validation. First we sample the signal uniformly with a low userdefined step (e.g., $30^{\circ}$ ). We assume that all the previously taken samples are sorted by their angle $\varphi_{v}$ and labeled by indices from 1 to $n$, when $n$ is a count of samples already taken. Now take sample $k \in\{1, \ldots, n\}$, draw a line through samples $k-1$ and $k+1$ and compare line value $u$ in position of the sample $k$ with the sample's value $v$. If the values differ more than a user-defined number of percents $t$ (e.g., 10\%) in any color channel, mark position $s_{-}$in the middle of sample $k-1$ and $k$ and position $s_{+}$in the middle of sample $k$ and $k+1$ as candidates for a new sample. Repeat the algorithm for all the samples. Note that the signal is circularly periodic.

When all the candidate positions of new samples are known, they can be measured and we can search for new candidate positions again. Algorithm stops when there are no candidate positions (due to a limited signal rate of innovation or measurement resolution) or after a defined number of iterations. When the samples are taken with sufficient

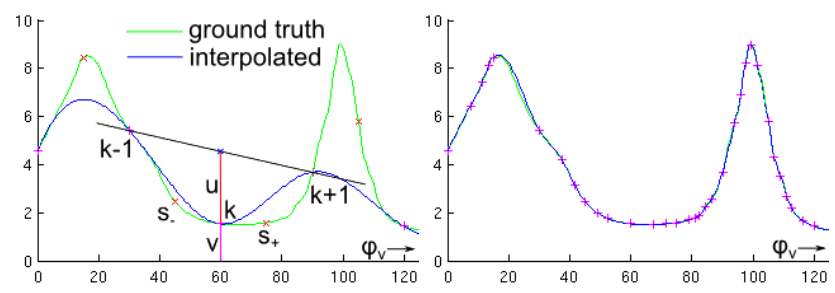

Figure 7. A principle of $1 \mathrm{D}$ adaptive sampling method. A shape after the first uniform step (5 samples, + ) and candidates $(\times)$ for the next step (left). Final state after six iterations (33 samp.) (right).

density every point on the slice can be interpolated very precisely, using e.g., a piece-wise cubic spline.

\subsection{Subspace Reconstruction from Slices}

To reconstruct an arbitrary point in a two-dimensional subspace from reconstructed slices, we use an adapted swept surface technique that uses two cross-section and two profile curves and constrains resulting minimal value (see Fig. 8). We tested also polynomial and spline interpolations, but they required either predefined rank or introduced disturbing artifacts.

Let the rectangle be axially aligned in a local coordinate system defined by axes $x$ and $y$ ranging from 0 to 1 . The

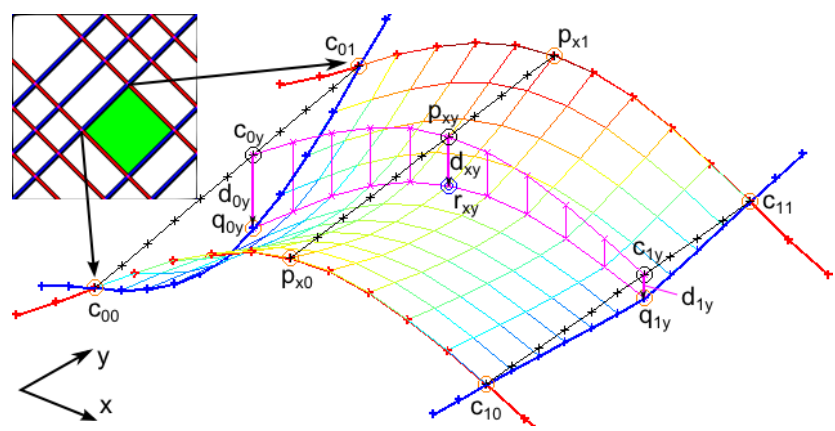

Figure 8. A scheme of 2D interpolation estimating interior points of a general rectangle from corner's and border's values.

interpolation requires knowledge of values of eight points to interpolate desired value $r_{x y}$ at $(x, y)$ inside the rectangle. The first four of them are values of corners of square $c_{x y}$, where $x, y \in\{0,1\}$. The next two values $p_{x 0}$ and $p_{x 1}$ are on the axial slices and the last two values $q_{0 y}$ and $q_{1 y}$ are on the diagonal slices. 
First, values $p_{x 0}$ and $p_{x 1}$ are linearly interpolated along axis $y$ yielding value $p_{x y}$

$$
p_{x y}=(1-y) * p_{x 0}+y * p_{x 1} .
$$

To compute value $r_{x y}$, the value $p_{x y}$ has to be compensated for a height difference introduced by a linear interpolation and values of the diagonal slices. Therefore, values $c_{0 y}$ and $c_{1 y}$ are also linearly interpolated along axis $y$ from $c_{00}, c_{01}$ and $c_{10}, c_{11}$ respectively

$$
\begin{aligned}
& c_{0 y}=(1-y) * c_{00}+y * c_{01}, \\
& c_{1 y}=(1-y) * c_{10}+y * c_{11} .
\end{aligned}
$$

The differences $d_{0 y}$ and $d_{1 y}$ between the linear interpolations of the corner values and values of the $x$-aligned slices are computed as $d_{0 y}=q_{0 y}-c_{0 y}$ and $d_{1 y}=q_{1 y}-c_{1 y}$. Finally, the height difference in the point $(x, y)$ is obtained with a linear interpolation of the differences in the axis $x$

$$
d_{x y}=(1-x) * d_{0 y}+x * d_{1 y} .
$$

The final value $r_{x y}$ is a sum of the value $p_{x y}$ and the difference $d_{x y}$ and its minimal value is constrained by a minimal value of $p_{x 0}, p_{x 1}, q_{0 y}$ and $q_{1 y}$

$$
r_{x y}=\max \left(p_{x y}+d_{x y}, \min \left(p_{x 0}, p_{x 1}, q_{0 y}, q_{1 y}\right)\right) .
$$

This processing is done separately for each color channel. It can be proven that changing $x, y$ axes interpolation order yields the same result.

\section{Results}

This section compares performance of the proposed interpolation method with uniform sampling using the same samples count. Due to the unavailability of accurate dense reference data and their very time-demanding measurement, we used only a single sample for our experiments. However, the selected sample has the strongest anisotropic behavior we have seen so far. Fig. 10 shows densely measured reference BRDF subspace (a), uniform sampling by means of $81 \times 81$ (b) and $151 \times 151$ (d) interpolated to the same azimuthal resolution as the reference using a barycentric interpolation. Fig. 10 (c) and (e) shows performance of the proposed interpolation method in suggested representation using 12 axial and 12 diagonal slices (f). As this grid provides a reasonable reconstruction quality for the tested complex material, we expect that it would be sufficient for most of the other materials. Note that the number of slices can be arbitrary. The $10 \times$ scaled difference images (see second row of Fig. 10 from the reference data show that the proposed parameterization and interpolation is able to achieve significantly better reconstruction of the original data using the same number of samples.

As the reconstruction of a single BRDF subspace is insufficient for any appearance visualization we measured eight additional subspaces at elevations $0^{\circ}, 30^{\circ}, 75^{\circ}$ and their combinations. These measurements were obtained using 6561 samples and their reconstructions using the proposed method are shown in Fig.9 (e).

Furthermore, we interpolated data at missing elevations using a four-dimensional Krig interpolation of spherical angles $\left(\theta_{i}, \varphi_{i}, \theta_{v}, \varphi_{v}\right)$ represented in a $0 \approx 2 \pi$ azimuthal continuity preserving directional parameterization [6]. Fig. 9 shows a comparison of ground truth measurements on sphere (a), with rendering using barycentrically interpolated $81 \times 81$ (b) and $151 \times 151$ (c) uniform samples. The result of the proposed data acquisition and interpolation method using 6561 adaptive samples is shown in (d). Note that

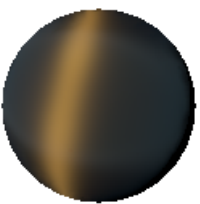

(a)

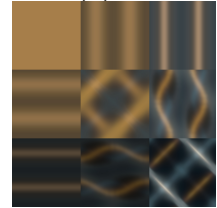

(e)

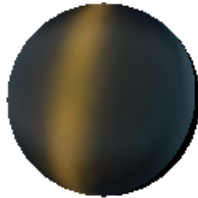

(b) $6561 \mathrm{~s}$.

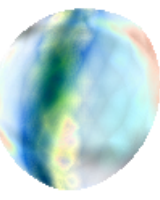

$3.8 / 8.5 / 29.5$

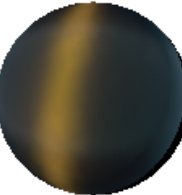

(c) $22801 \mathrm{~s}$.

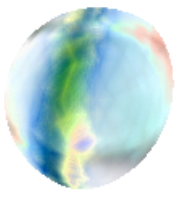

$3.7 / 8.0 / 30.1$

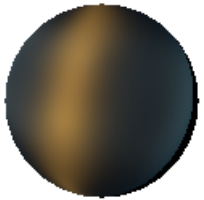

(d) $6561 \mathrm{~s}$.

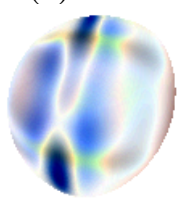

$3.8 / 7.6 / 30.6$
Figure 9. Per-pixel ground truth measurements on a sphere (a) compared with barycentrically interpolated uniform measurements (b), (c) and with the proposed interpolation from sparse measurements (d). Below are the difference values in: CIE $\triangle \mathrm{E} / \mathrm{RMSE} / \mathrm{PSNR}[\mathrm{dB}]$ as well as the $10 \times$ difference images. Measured and densely reconstructed nine subspaces used for our method visualization (e).

although the reconstruction quality gain might look small, we believe that it can be considerably improved by the proposed adaptive measurement at additional elevations, i.e., without relying on the missing subspaces interpolation.

The reconstruction of the BRDF subspace $(720 \times 720$ pixels) adaptively distributed in 24 slices takes typically 1-2 seconds regardless the sample count at Intel Xeon $2.7 \mathrm{GHz}$ using a single core and non-optimized Matlab implementation. Note that the measured sparse BRDF as well as its densely sampled subspaces are publicly available for research purposes in UTIA BTF Database $[5]^{1}$

\section{Conclusions}

We presented a sparse BRDF data representation and interpolation methods that outperform in reconstruction quality uniform sampling using the same count of samples. The proposed sliced parametrization allows for fast, continuous acquisition at fixed elevations of camera and light, and fast, robust reconstruction of non-measured values at arbitrary

\footnotetext{
${ }^{1}$ http://btf.utia.cas.cz
} 
(a) reference 518400 samples

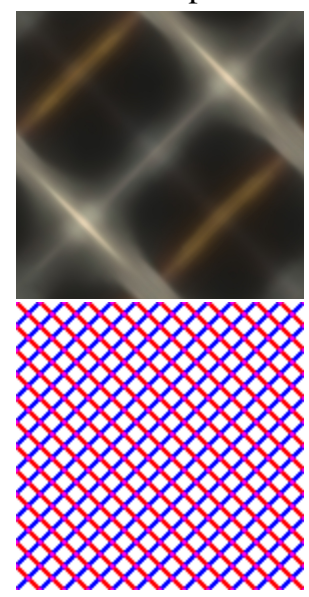

(f) (b) uniform sampl. 576 samples

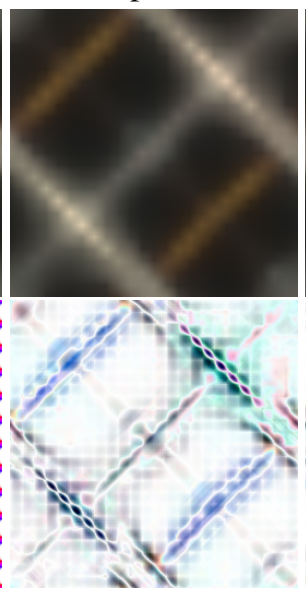

$1.7 / 4.6 / 34.9 / 0.99$ (c) slices interp. 576 samples

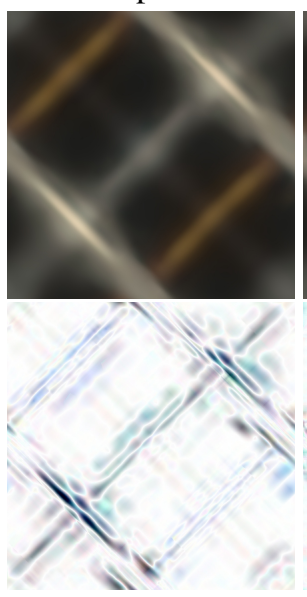

$0.9 / 3.2 / 38.2 / 0.99$ (d) uniform sampl. 2304 samples

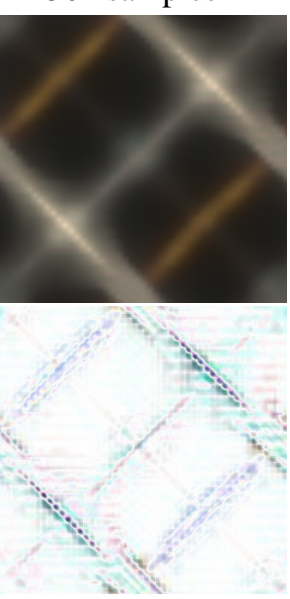

$0.8 / 2.1 / 41.6 / 0.99$ (e) slices interp. 2304 samples

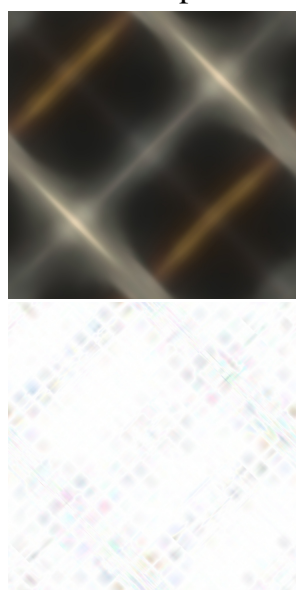

$0.3 / 0.6 / 52.2 / 1.00$

Figure 10. The reference BRDF subspace $720 \times 720$ (a) compared with the same size barycentric interpolations from two densities of uniform sampling $24 \times 24$ (b) and $48 \times 48$ (d), and the proposed interpolation from the sparse representation using the same samples count (c) and (e), using thresholds $t=0.47$ and $t=0.054$, respectively. Below are the $10 \times$ difference images and difference values in: CIE $\Delta \mathrm{E} / \mathrm{RMSE} / \mathrm{PSNR}[\mathrm{dB}]$ / SSIM. Bottom-left: the proposed uniform placement of measured slices.

resolution. The method's accuracy is given by a used number of slices and their adaptive sampling density specified by maximal allowed error. We believe that the proposed data-driven sampling together with the robust reconstruction performance represents an initial framework for intelligent adaptive sampling methods of view- and illuminationdependent material appearance.

Acknowledgements - This work has been supported by the Czech Science Foundation grants P103/11/0335, P202/12/2413 and EC Marie Curie ERG 239294.

\section{References}

[1] K. Dana. BRDF/BTF measurement device. In ICCV 2001, volume 2, pages 460-466, July 2001.

[2] J. Filip and R. Vávra. Fast method of sparse acquisition and reconstruction of view and illumination dependent datasets. To appear in Computer and Graphics, page 12, 2013.

[3] M. Fuchs, V. Blanz, H. P. Lensch, and H.-P. Seidel. Adaptive sampling of reflectance fields. ACM Trans. Graph., 26(2):118, June 2007.

[4] A. Ghosh, S. Achutha, W. Heidrich, and M. O'Toole. BRDF acquisition with basis illumination. ICCV 2007, 0:1-8.

[5] M. Haindl, J. Filip, and R. Vávra. Digital material appearance: the curse of tera-bytes. ERCIM News, No. 90, pages 49-50, 2012.

[6] V. Havran, J. Filip, and K. Myszkowski. Bidirectional texture function compression based on the multilevel vector quantization. Comp. Graph. Forum, 29(1):175-190, 2010.

[7] M. Holroyd, J. Lawrence, and T. Zickler. A coaxial optical scanner for synchronous acquisition of 3D geometry and surface reflectance. ACM Tran. Graph., 29:1-12, 2010.

[8] A. Höpe, T. Atamas, D. Hünerhoff, S. Teichert, and K.O. Hauer. Argon3: 3D appearance robot-based goniore- flectometer at PTB. Review of Scientific Instruments, 83(4):045102, 2012.

[9] J. Lawrence, S. Rusinkiewicz, and R. Ramamoorthi. Adaptive numerical cumulative distribution functions for efficient importance sampling. In Eurographics Symposium on Rendering, pages 11-20, 2005.

[10] J. Lu and J. Little. Reflectance function estimation and shape recovery from image sequence of a rotating object. In ICCV 1995, pages 80-86, 1995.

[11] S. R. Marschner, S. H. Westin, E. P. F. Lafortune, and K. E. Torrance. Image-based bidirectional reflectance distribution function measurement. Applied Optics, 39:2592-2600, 2000.

[12] W. Matusik, H. Pfister, M. Brand, and L. McMillan. A datadriven reflectance model. ACM Trans. Graph., 22(3):759769, 2003.

[13] G. Müller. Data-Driven Methods for Compression and Editing of Spatially Varying Appearance. Dissertation, Universität Bonn, Dec. 2009.

[14] A. Ngan, F. Durand, and W. Matusik. Experimental analysis of BRDF models. Eurographics Symposium on Rendering 2005, 2:117-126, 2005.

[15] F. Nicodemus, J. Richmond, J. Hsia, I. Ginsburg, and T. Limperis. Geometrical considerations and nomenclature for reflectance. NBS Monograph 160, National Bureau of Standards, U.S. Dept. of Com., pages 1-52, 1977.

[16] J. Robinson. Image coding with ridge and valley primitives. IEEE Trans. on Communic., 43(6):2095-2102, 1995.

[17] M. Sattler, R. Sarlette, and R. Klein. Efficient and realistic visualization of cloth. In Eurographics Symposium on Rendering 2003, pages 167-178, 2003.

[18] S. K. Thompson and G. A. F. Seber. Adaptive Sampling. John Wiley and sons, New York, 1996. 